\title{
Optical Spectroscopic Monitoring Observations of a Young Binary Z CMa
}

\author{
Hinako Akimoto, Yoichi Itoh \\ Nishi-Harima Astronomical Observatory, Center for Astronomy, University of Hyogo, Sayo, Hyogo, Japan \\ Email: yitoh@nhao.jp
}

How to cite this paper: Akimoto, $\mathrm{H}$. and Itoh, Y. (2021) Optical Spectroscopic Monitoring Observations of a Young Binary $\mathrm{Z}$ CMa. International Journal of Astronomy and Astrophysics, 11, 406-421. https://doi.org/10.4236/ijaa.2021.113019

Received: July 30, 2021

Accepted: September 10, 2021

Published: September 13, 2021

Copyright $\odot 2021$ by author(s) and Scientific Research Publishing Inc. This work is licensed under the Creative Commons Attribution International License (CC BY 4.0).

http://creativecommons.org/licenses/by/4.0/ (c) (i) Open Access

\begin{abstract}
The results of optical spectroscopic monitoring observations of a young binary system, Z CMa, are presented in this study. Z CMa consists of a Herbig Be star and an FU Orionis object, and it shows irregular light variation in the quiescent phase and exploding brightening in the outburst phase. Medium-resolution spectra were obtained on 21 nights between 2015 and 2019 using the Nayuta telescope in Japan. We also used five high-resolution spectra, obtained between 2008 and 2011, with the Keck Telescope. During the outburst phase, the intensity of the He I absorption line increased with an increase in the luminosity of the system. Because the He I absorption line is a characteristic feature of an early-type star, we considered that the outbursts were caused by the Herbig Be star. The equivalent widths of the [O I] line decreased with an increase in the luminosity of the system. We claim steady mass loss at the rate of $10^{-5.5} \mathrm{M}_{\odot} \cdot \mathrm{yr}^{-1}$. The intensities of the $\mathrm{H} \alpha$ and $\mathrm{Fe}$ II emission lines did not change during the outbursts. However, the line intensities increased with an increase in the luminosity of the system in the quiescent phase. We consider that those lines are attributed to the FU Orionis object and the light variations in the quiescent phase were caused by the FU Orionis object.
\end{abstract}

\section{Keywords}

Stars:Pre-Main Sequence, Stars:Variables:T Tauri, Herbig Ae/Be, Techniques:Spectroscopic

\section{Introduction}

A young stellar object (YSO) gains its weight by accretion of surrounding materials through a circumstellar disk. Steady mass accretion rate of an order of $10^{-8}$ $\mathrm{M}_{\odot} \cdot \mathrm{yr}^{-1}$ has been proposed for a solar-mass YSO [1]. Mass accretion from the 
circumstellar disk to the stellar surface lasts $10^{6} \mathrm{yr}$. Thus, the steady mass accretion is insufficient for the star to get the final mass. One of the phenomena that may solve this problem is the rapid increase in the luminosity observed for YSOs. V1057 Cyg increased its luminosity by more than 5 magnitudes in 1969 [2], and this luminosity increase was interpreted as a temporary increase in the mass accretion rate by a factor of 1000 . An object that shows a sudden increase in luminosity and maintains the bright phase for more than a decade is called an FU Orionis object. An object that experiences a brightening phase for a shorter period than an FU Orionis object is called an EXor.

$\mathrm{Z} \mathrm{CMa}$ is an YSO that shows irregular light variations. In the past twenty years, $Z$ CMa has repeatedly exhibited 0.5 - 1.5 magnitude changes in the optical wavelengths [3]. In addition, $\mathrm{Z} \mathrm{CMa}$ has often exhibited sudden increases in brightness. The amplitudes had approximately 2-magnitude in the $V$-band and the brightening phase lasted for $\sim 100$ days with an interval of hundreds of days. These EXor-like outbursts occurred in 2000, 2004, 2008, 2011, 2015, and 2016 [4] [5].

Near-infrared speckle interferometry resolved two components of the binary [6] [7]. It is indicated that the south-eastern component dominates the visual and ultraviolet parts of the spectral energy distribution (SED). Although this component has not undergone a large outburst in the last century, it has been identified as an FU Orionis object [8]. This star has a spectral type of F, a mass of $3 \mathrm{M}_{\odot}$, a radius of $7 \mathrm{R}_{\odot}$, and an age of $3 \times 10^{5} \mathrm{yr}$. The north-western component dominates the infrared parts of the SED. It was identified as a Herbig Be star [9], surrounded by a dust cocoon [3]. Its mass is $16 \mathrm{M}_{\odot}$, radius is $7.6 \mathrm{R}_{\odot}$, and age is estimated to be $3 \times 10^{5} \mathrm{yr}[3]$.

The large scale picture that the Herbig Be star is surrounded by a dust cocoon of variable geometry and the whole system is surrounded by an infalling envelope is widely supported by many observational results (e.g. [10]). Optical spectropolarimetry of Z CMa was carried out in 1991-1992 [9]. At that time, Z CMa was at $V \sim 9.6-9.8$ mag. Polarization of $1 \%-2 \%$ in the continuum and up to $6 \%$ in the emission lines were detected. It was argued that the Herbig Be star was the emission line source and it was mostly obscured by an asymmetrical dust cocoon. The outburst was attributed to the light variation of the Herbig Be star. Another spectropolarimetric observation was carried out in the outburst phase in 2008 [4]. The polarization angles of the emission lines were perpendicular to the large-scale jet. It was claimed that the outburst was the phenomenon that more light from the Herbig Be star reached the observer through a widened cavity.

JHKL-band photometry of the individual components was carried out by using the adaptive optics system [11]. Observations showed that the flux of the Herbig Be star increased significantly for wavelengths shorter than $10 \mu \mathrm{m}$ during the outburst. It was concluded that the outburst was caused by the Herbig Be star, and this was confirmed through spectroscopy. During the outburst in 1987, the flux of the Balmer emission lines and the Ca II $\mathrm{K}$ emission line, as well as the 
brightness of Z CMa, increased. Moreover, many metallic lines, such as Fe II, $\mathrm{Cr}$ II, and Ti II, changed from absorption to emission lines [12]. These phenomena were interpreted as the outbursts caused by the Herbig Be star.

We have carried out a series of optical spectroscopy of Z CMa. We also investigated the relationship between the line intensities obtained from spectroscopy and the luminosity obtained from broad-band photometry.

\section{Observations and Data Reduction}

We conducted spectroscopic observations of $\mathrm{Z} \mathrm{CMa}$ on 21 nights between October 2015 and November 2019 by using the medium- and low-dispersion long-slit spectrograph (MALLS) mounted on the $2.0 \mathrm{~m}$ Nayuta telescope at Nishi-Harima Astronomical Observatory, Japan. Using a grating of 1800 lines $/ \mathrm{mm}$ and a 0.8 " slit, we obtained spectra with a wavelength resolution of $\sim 10,000$ between $6280 \AA$ and $6720 \AA$. The exposure time ranged from $300 \mathrm{~s}$ to $1200 \mathrm{~s}$, and 1 - 4 spectra were obtained each night. Flat frames and comparison frames were acquired by using a halogen lamp and an Fe-Ne-Ar lamp in the instrument, respectively. Dark frames were also obtained. Details of the observations are presented in Table 1.

We also used data obtained by employing the high-resolution echelle spectrometer (HIRES) of the Keck telescope. The data between 2008 and 2011 were obtained from the archive system. The spectral resolution was $\sim 48,000$ between $5700 \AA$ and $7150 \AA$. We obtained object frames, bias frames, flat frames, and Th-Ar lamp comparison frames. Details of the observations are also listed in Table 1.

The MALLS data were reduced using the IRAF software. The average count of the overscan region was subtracted from the entire region. Ten dark frames were combined with the median, and the combined dark image was subtracted from the object and flat frames. The normalized flat image was used to correct the sensitivity of each CCD element. We identified approximately 50 emission lines of the Fe-Ne-Ar lamp in the comparison image and established a relationship between the column number of the pixel and the wavelengths. The column number of the pixel of the object frame was converted into the wavelength using this relationship. We also corrected the spatial distortion of the object image using the comparison image. We then fitted background emission as a function of the columns of the image and subtracted it from the entire region. We extracted a one-dimensional spectrum from a two-dimensional spectrum in the object frame. The extracted spectrum was binned by the slit width. The Doppler shift due to the orbital motion of the Earth was corrected. We combined the spectra acquired each night. Finally, we normalized the continuum level of the spectrum.

Image processing of the HIRES data was performed through a pipeline called Mauna Kea Echelle Extraction in addition to IRAF. Each component of the binary system was not resolved either in the MALLS data or in the HIRES data. 
Table 1. Observation date, integration time, and $V$-band magnitude of the object.

\begin{tabular}{|c|c|c|c|c|}
\hline Date (JST) & JD $(-2,450,000)$ & Integ. Time (s) & $V$-mag & Telescope \\
\hline $2008-12-03$ & 4804 & 60 & 8.7 & Keck (PI: Dahm) \\
\hline $2008-12-04$ & 4805 & 60 & 8.7 & Keck (PI: Dahm) \\
\hline $2008-12-17$ & 4818 & 60 & 8.5 & Keck (PI: Hireseng) \\
\hline $2010-11-14$ & 5515 & 200 & 8.5 & Keck (PI: Herbig) \\
\hline 2011-11-18 & 5884 & 180 & 10.1 & Keck (PI: Reipurth) \\
\hline $2015-10-13$ & 7309 & 1800 & 9.95 & Nayuta \\
\hline $2015-10-31$ & 7327 & 1800 & 9.82 & Nayuta \\
\hline $2015-11-29$ & 7356 & 1800 & 9.61 & Nayuta \\
\hline $2015-12-01$ & 7358 & 1800 & 9.61 & Nayuta \\
\hline 2016-01-10 & 7398 & 1800 & 8.14 & Nayuta \\
\hline $2016-01-12$ & 7400 & 1800 & 8.14 & Nayuta \\
\hline $2016-01-13$ & 7401 & 1800 & 8.19 & Nayuta \\
\hline $2016-01-16$ & 7404 & 1800 & 8.19 & Nayuta \\
\hline 2016-03-03 & 7451 & 1800 & 8.53 & Nayuta \\
\hline 2016-03-11 & 7459 & 1800 & 8.68 & Nayuta \\
\hline 2016-04-05 & 7484 & 1800 & 9.20 & Nayuta \\
\hline $2016-10-13$ & 7675 & 1800 & 8.71 & Nayuta \\
\hline $2016-10-20$ & 7682 & 1800 & 8.88 & Nayuta \\
\hline $2016-11-03$ & 7696 & 1800 & 9.36 & Nayuta \\
\hline 2016-11-08 & 7701 & 1800 & 9.48 & Nayuta \\
\hline $2016-11-11$ & 7704 & 1800 & 9.50 & Nayuta \\
\hline $2016-11-17$ & 7710 & 1800 & 9.74 & Nayuta \\
\hline $2016-11-21$ & 7714 & 1800 & 9.72 & Nayuta \\
\hline $2018-01-23$ & 8142 & 1800 & 10.34 & Nayuta \\
\hline 2019-01-15 & 8499 & 1800 & 10.01 & Nayuta \\
\hline 2019-01-18 & 8502 & 1800 & 9.93 & Nayuta \\
\hline
\end{tabular}

\section{Results}

Figure 1 shows an example of the spectra obtained with MALLS. Emission lines of [O I], Fe II, and $\mathrm{H} \alpha$, as well as the He I absorption line, appear in the spectra. The equivalent widths of these lines were measured by applying direct integration using IRAF (Table 2).

Figure 2 shows the light curve of $\mathrm{Z} \mathrm{CMa}$. We considered that the light variation is a combination of a small amplitude about 0.5 magnitude around 10 magnitude and a large amplitude about 2 magnitude around 9 magnitude. We classified the object as being in the quiescent phase when it is fainter than 9.6 magnitude in the $V$-band and the outburst phase when it is brighter than 9.6 magnitude in the $V$-band. 


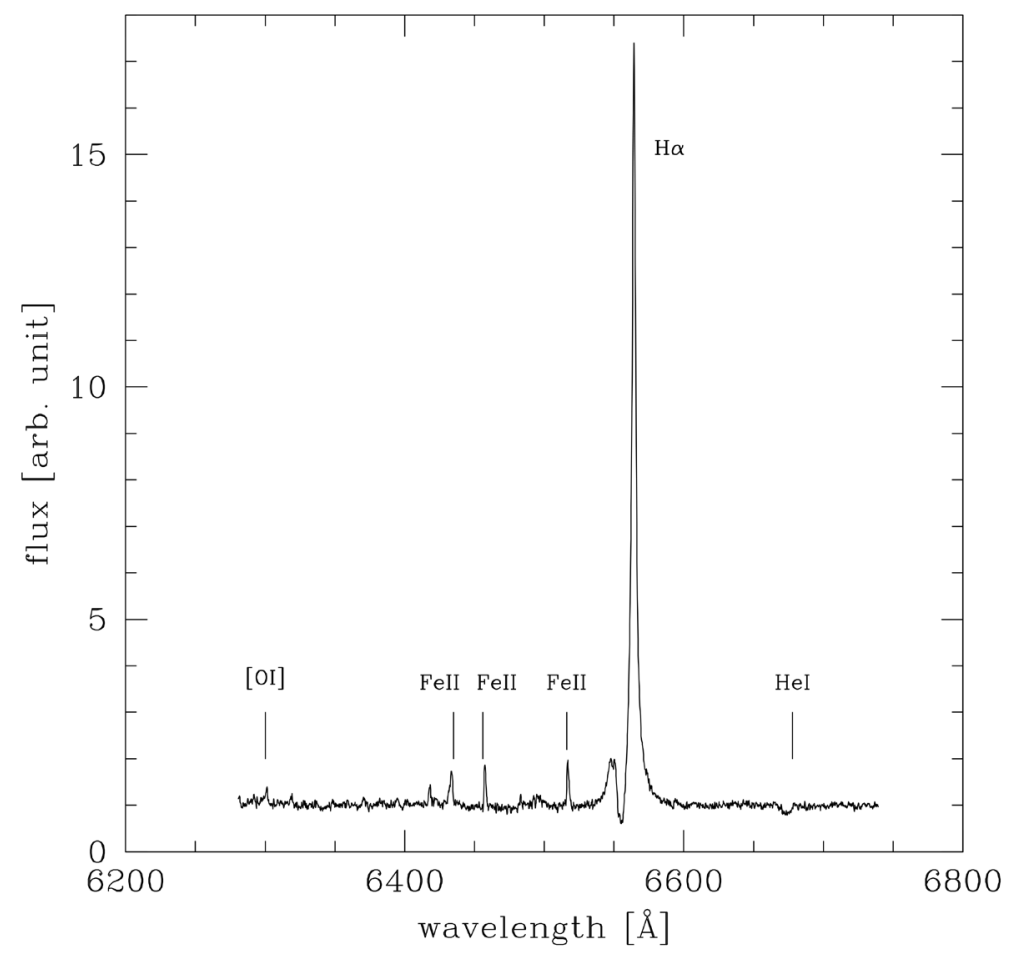

Figure 1. Optical spectrum of Z CMa obtained on October 20, 2016 with MALLS. The spectral flux is normalized with the continuum flux. The prominent features in the spectrum are the $\mathrm{H} \alpha$ emission line with a blueshifted absorption component, [O I] forbidden line (6300 $)$, Fe II emission lines (6435 ̊, $6456 \AA$, and $6516 \AA$ ), and He I absorption line (6678 $)$.

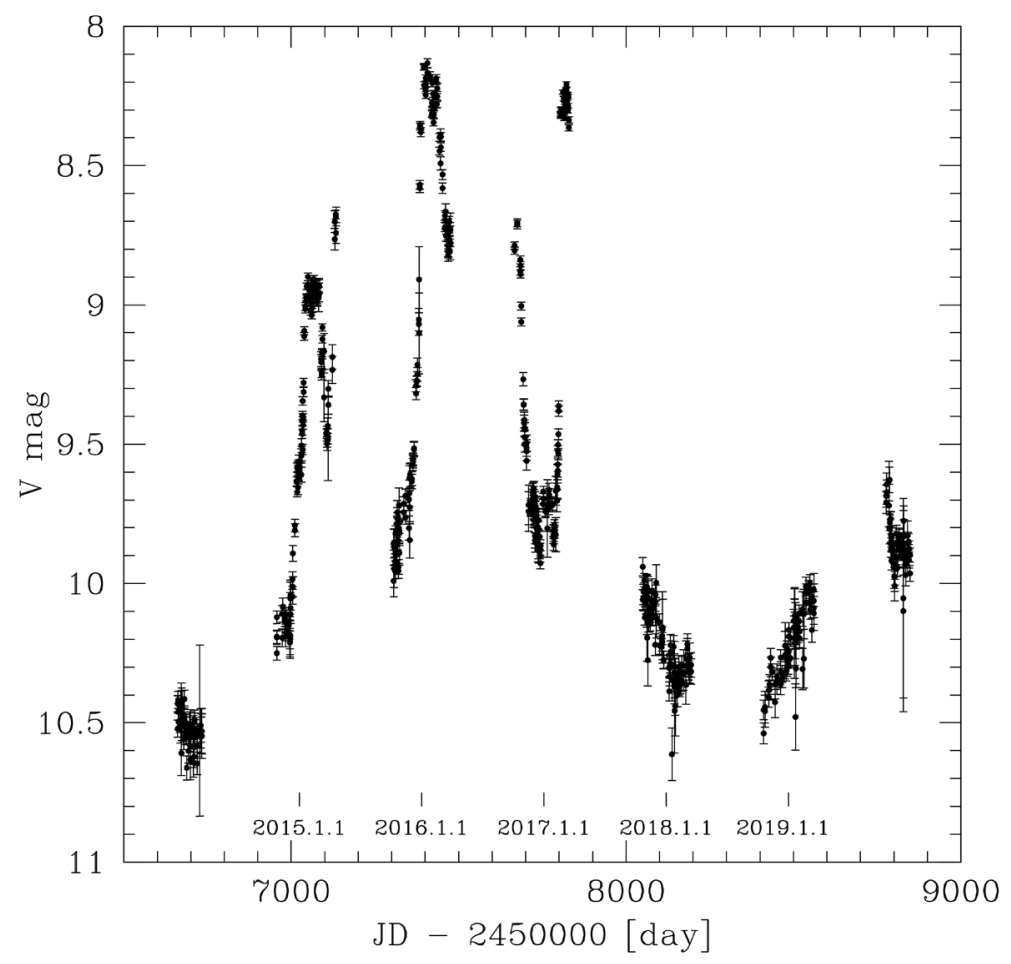

Figure 2. Light curve of $\mathrm{Z} \mathrm{CMa}$. The $V$-band magnitudes were obtained from the database of Kiso/Kyoto Wide-field Survey [13]. Outbursts are observed during February 2015, February 2016, and March 2017, with the amplitude of approximately 2 magnitudes. 
Table 2. Equivalent widths of the prominent features. The unit is $\AA$.

\begin{tabular}{|c|c|c|c|c|c|c|}
\hline Date (JST) & $\mathrm{He} \mathrm{I}$ & $\mathrm{H} \alpha$ & [O I] & Fe II 6435 & Fe II 6456 & Fe II 6516 \\
\hline $2008-12-03$ & 0.96 & $-63.2_{+0.4}^{-0.5}$ & $-1.60_{+0.21}^{-0.19}$ & -2.03 & -2.00 & -2.46 \\
\hline 2008-12-04 & 0.85 & $-76.5 \pm 0.4$ & $-1.37_{+0.14}^{-0.19}$ & -2.06 & -2.08 & -2.57 \\
\hline $2008-12-17$ & & $-38.7 \pm 0.3$ & $-1.18_{+0.05}^{-0.08}$ & -1.36 & -1.24 & -1.48 \\
\hline 2010-11-14 & 1.27 & $-38.5_{+0.3}^{-0.4}$ & $-1.51 \pm 0.13$ & -1.85 & -2.11 & -2.19 \\
\hline $2011-12-18$ & 0.22 & $-85.9_{+0.6}^{-0.7}$ & $-3.38_{+0.23}^{-0.25}$ & -1.42 & -1.58 & -1.71 \\
\hline $2015-10-13$ & 0.20 & $-156.3_{+0.9}^{-1.0}$ & $-3.03_{+0.27}^{-0.29}$ & $-3.44_{+0.14}^{-0.15}$ & $-3.27 \pm 0.09$ & $-3.48_{+0.11}^{-0.12}$ \\
\hline $2015-10-14$ & 0.21 & $-167.7 \pm 0.9$ & $-3.33_{+0.28}^{-0.29}$ & $-3.56_{+0.13}^{-0.15}$ & $-3.42 \pm 0.09$ & $-3.43 \pm 0.11$ \\
\hline $2015-10-31$ & 0.24 & $-132.2 \pm 1.0$ & $-3.10_{+0.31}^{-0.32}$ & $-2.90_{+0.15}^{-0.16}$ & $-2.48_{+0.10}^{-0.11}$ & $-2.89_{+0.12}^{-0.13}$ \\
\hline $2015-11-29$ & 0.14 & $-146.8_{+1.0}^{-1.1}$ & $-2.52_{+0.30}^{-0.32}$ & $-3.46 \pm 0.17$ & $-3.31 \pm 0.11$ & $-3.27 \pm 0.13$ \\
\hline $2015-12-01$ & 0.13 & $-150.6 \pm 1.1$ & $-3.13 \pm 0.36$ & $-3.48 \pm 0.18$ & $-3.30 \pm 0.12$ & $-3.46_{+0.14}^{-0.15}$ \\
\hline 2016-01-10 & 1.74 & $-44.6_{+1.5}^{-1.4}$ & $-0.54_{+0.24}^{-0.33}$ & $-1.58_{+0.20}^{-0.22}$ & $-1.35_{+0.12}^{-0.13}$ & $-1.33 \pm 0.13$ \\
\hline 2016-01-12 & 1.36 & $-50.0 \pm 0.6$ & $-0.67_{+0.15}^{-0.17}$ & $-1.51_{+0.08}^{-0.09}$ & $-1.34 \pm 0.05$ & $-1.40 \pm 0.06$ \\
\hline 2016-01-13 & 1.32 & $-52.4 \pm 0.7$ & $-0.88_{+0.19}^{-0.22}$ & $-1.51_{+0.10}^{-0.11}$ & $-1.35 \pm 0.06$ & $-1.50 \pm 0.07$ \\
\hline 2016-01-16 & 1.09 & $-49.2_{-0.6}^{-0.7}$ & $-0.79_{+0.15}^{-0.18}$ & $-1.37_{+0.09}^{-0.10}$ & $-1.17 \pm 0.05$ & $-1.26 \pm 0.06$ \\
\hline 2016-03-03 & 1.40 & $-66.6_{+0.6}^{-0.7}$ & $-1.52_{+0.19}^{-0.20}$ & $-1.85_{+0.09}^{-0.10}$ & $-1.36_{+0.05}^{-0.06}$ & $-1.68_{+0.06}^{-0.08}$ \\
\hline 2016-03-11 & 0.86 & $-84.2_{+3.2}^{-3.1}$ & $-1.67_{+0.73}^{-0.88}$ & $-2.37_{+0.41}^{-0.45}$ & $-1.58_{+0.27}^{-0.30}$ & $-2.15_{+0.28}^{-0.35}$ \\
\hline 2016-04-05 & 0.64 & $-111.2 \pm 1.0$ & $-1.94_{+0.30}^{-0.33}$ & $-2.81 \pm 0.17$ & $-2.45 \pm 0.11$ & $-2.75_{+0.11}^{-0.14}$ \\
\hline $2016-10-13$ & 1.00 & $-59.1 \pm 1.1$ & $-1.78_{+0.32}^{-0.35}$ & $-1.78_{+0.17}^{-0.18}$ & $-1.43_{+0.08}^{-0.09}$ & $-1.54_{+0.12}^{-0.13}$ \\
\hline $2016-10-20$ & 1.33 & $-80.8 \pm 2.4$ & $-1.97_{+0.66}^{-0.75}$ & $-2.15_{+0.35}^{-0.38}$ & $-1.36_{+0.13}^{-0.15}$ & $-1.84_{+0.23}^{-0.29}$ \\
\hline 2016-11-03 & 0.16 & $-121.6 \pm 1.0$ & $-2.36_{+0.30}^{-0.32}$ & $-3.35 \pm 0.16$ & $-3.15 \pm 0.11$ & $-3.11_{+0.12}^{-0.13}$ \\
\hline 2016-11-08 & 0.13 & $-136.5_{+1.1}^{-1.0}$ & $-2.83_{+0.31}^{-0.32}$ & $-3.54 \pm 0.16$ & $-3.35 \pm 0.10$ & $-3.43 \pm 0.12$ \\
\hline 2016-11-11 & 0.12 & $-133.0 \pm 0.9$ & $-2.90_{+0.28}^{-0.29}$ & $-3.43 \pm 0.15$ & $-3.16 \pm 0.10$ & $-3.28 \pm 0.11$ \\
\hline 2016-11-17 & 0.13 & $-142.4_{+1.0}^{-0.9}$ & $-3.66 \pm 0.29$ & $-3.53 \pm 0.15$ & $-3.37 \pm 0.10$ & $-3.44 \pm 0.11$ \\
\hline $2016-11-21$ & 0.06 & $-136.0_{+1.1}^{-1.0}$ & $-3.53_{+0.32}^{-0.33}$ & $-3.42_{+0.16}^{-0.17}$ & $-3.11_{+0.11}^{-0.12}$ & $-2.85 \pm 0.12$ \\
\hline $2018-01-23$ & 0 & $-119.9 \pm 2.5$ & $-3.36_{+0.62}^{-0.71}$ & $-1.26_{+0.24}^{-0.33}$ & $-0.70_{+0.15}^{-0.20}$ & $-1.12_{+0.20}^{-0.24}$ \\
\hline $2019-01-15$ & 0.22 & $-130.1 \pm 0.9$ & $-3.14_{+0.26}^{-0.27}$ & $-2.95_{+0.10}^{-0.11}$ & $-2.83_{+0.07}^{-0.08}$ & $-3.06 \pm 0.10$ \\
\hline 2019-01-18 & 0.07 & $-139.1_{+1.1}^{-1.0}$ & $-4.03 \pm 0.32$ & $-3.61 \pm 0.16$ & $-3.28 \pm 0.11$ & $-3.37_{+0.12}^{-0.13}$ \\
\hline
\end{tabular}

\section{Discussion}

\subsection{He I}

The He I absorption line has been detected in many Herbig Ae/Be stars. A Herbig Ae/Be star, V645 Cyg, has blueshifted absorption features in the He I absorption line [14]. The blueshifted absorptions are indicative of a high-velocity out- 
flow. High-dispersion spectra of Z CMa were acquired between 1985 and 1988 [12]. At that time, the He I line showed a broad absorption feature.

In our observations, the He I line showed a weak emission feature during the quiescent phase and a blueshifted broad absorption feature during the outburst phase (Figure 3). The equivalent widths of the emission component were between 0 and $-0.7 \AA$, and those of the absorption component were between 0 and $1.7 \AA$. Figure 4 shows the relationship between the equivalent widths of the $\mathrm{He} \mathrm{I}$ absorption component and the $V$-band magnitudes. The least square fit of the points for $V<9.6 \mathrm{mag}$ is also shown. If the line intensity is constant, the equivalent widths would be small when the object is bright. However, in the outburst phase, the equivalent widths of the absorption component increased with an increase in the luminosity of the object. This result indicates that the line intensity increased during the outburst phase and the source of the He absorption feature and that of the outbursts are the same object. Because the He I absorption

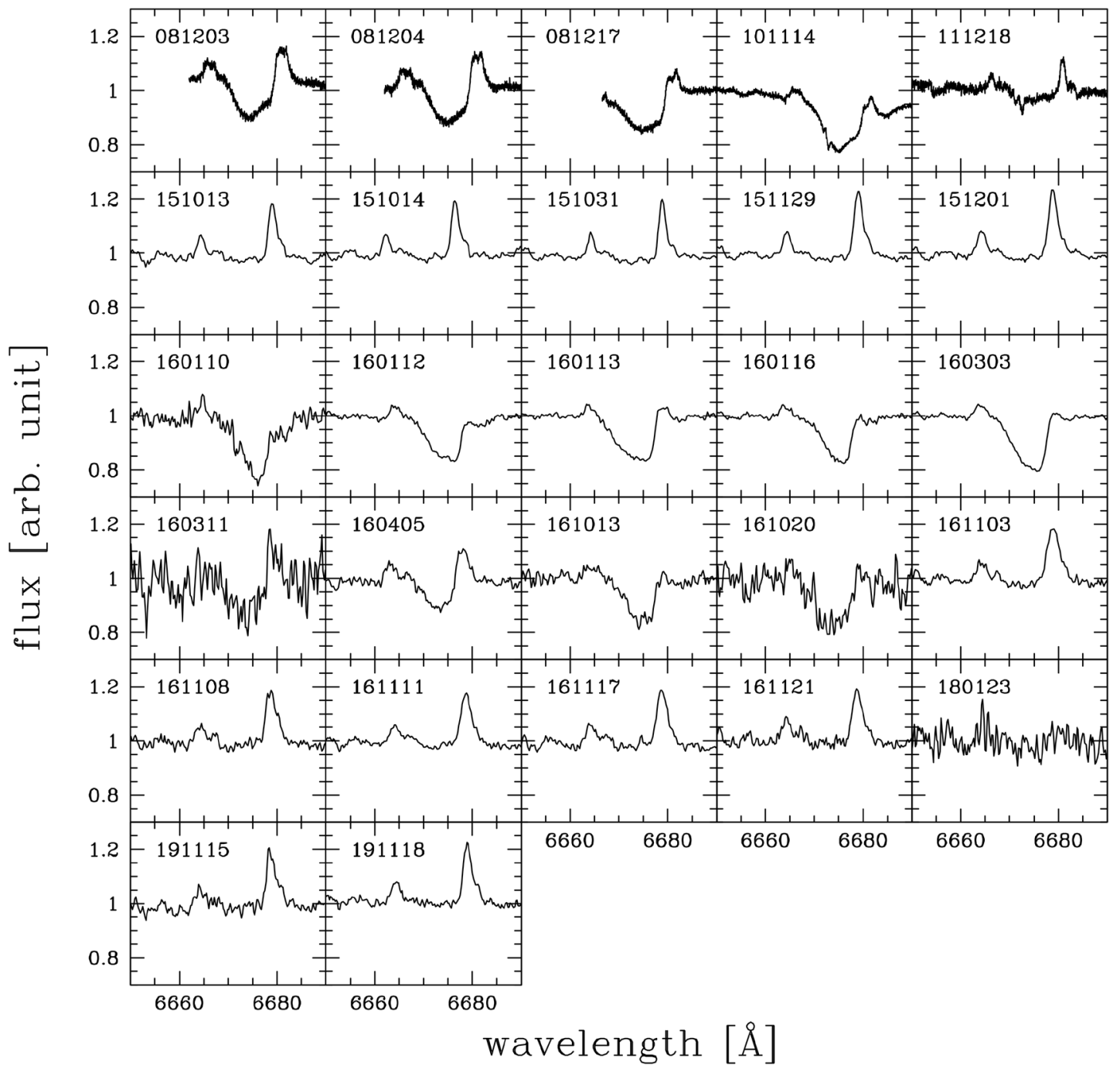

Figure 3. Line profiles of the He I emission and absorption features. The rest wavelength of the He I line is $6678 \AA$. The emission at $6663 \AA$ is attributed to Fe. 


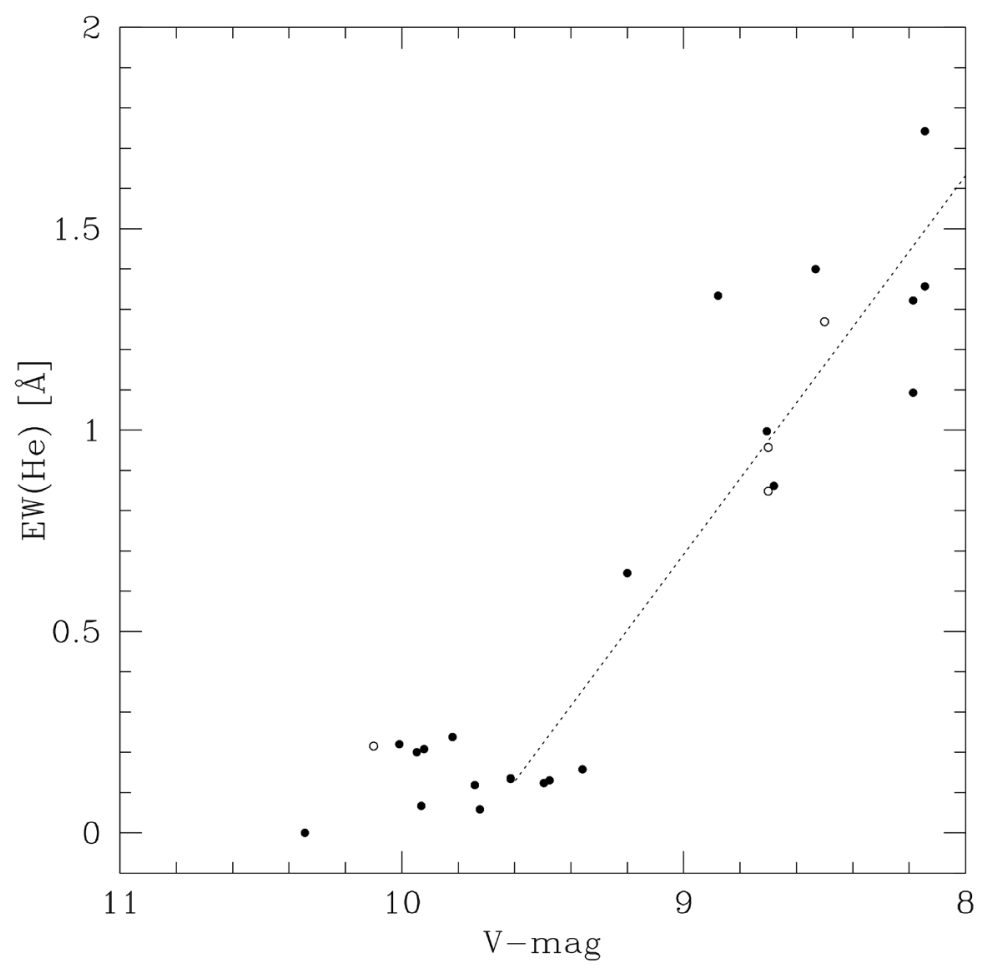

Figure 4. Relationship between the equivalent widths of the absorption feature of the $\mathrm{He}$ I line and the $V$-band magnitude. The filled circles represent the measurements of the MALLS spectra, and the open circles represent those of the HIRES spectra. The dotted line represents the least square fit of the points for $V<9.6$ mag. Its correlation coefficient is 0.89 .

feature originates from the Herbig Be star, we considered it to be the source of the outbursts.

\section{2. [O I]}

It is well-known that the [O I] emission traces a jet. [O I] narrow-band images of $\mathrm{Z}$ CMa were obtained with an angular resolution of $0.03 \mathrm{arcsec}$ [15], and a collimated jet emanating from the FU Orionis object was detected. The mass loss rate of this jet was estimated to be $10^{-8}$ to $10^{-6} \mathrm{M}_{\odot} \cdot \mathrm{yr}^{-1}$.

In the optical spectra of Z CMa, the [O I] emission line showed a double peak feature with peaks at $6291.0 \AA$ and $6300.6 \AA$ (Figure 5). The separation of the peaks corresponded to $440 \mathrm{~km} \cdot \mathrm{s}^{-1}$ in radial velocity. We measured the equivalent width of the [O I] emission line including two peaks. Figure 6 shows the relationship between the equivalent widths and the $V$-band magnitudes of the object. The equivalent widths of the [O I] emission line decreased with an increase in the luminosity of the object. We constructed a simple model such that the continuum flux increased without any change in the intensity of the [O I] emission line. When the intensity of the continuum light increased by a factor of $\alpha$, the equivalent width of the line decreased by a factor of $\alpha$. The coefficient of determination between the model and the observed equivalent widths, $R^{2}$, is 0.83 . The equivalent widths of the [O I] line are small when the star outbursts. Hence, 


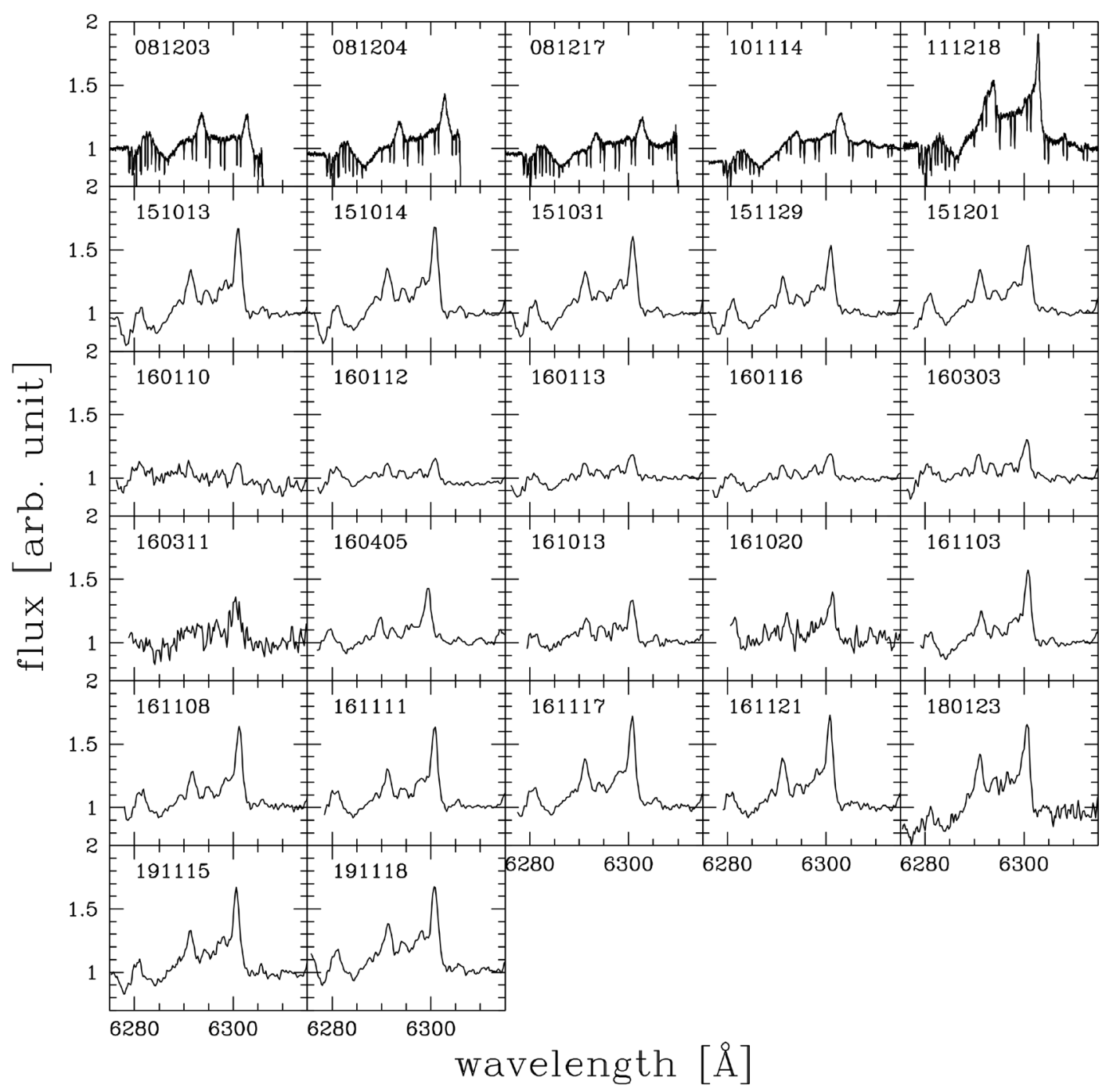

Figure 5. Profiles of the [O I] line. Two peaks appear at $6291.0 \AA$ and $6300.6 \AA$. In the HIRES spectra shown at the top of the figure, telluric absorption lines appear.

we concluded that the [O I] emission line was veiled when the outbursts occurred.

It is claimed that the blue peak did not exist until 2000 but grew significantly in 2002 [3]. The equivalent widths of the blue side and red side of the [O I] emission line, separated by the border at $6295.8 \AA$, are roughly correlated. We considered that the two peaks originated from the same star.

The mass loss rate was estimated from the [O I] luminosity. We considered $1150 \mathrm{pc}$ as the distance to $\mathrm{Z} \mathrm{CMa}$. We assumed constant $V$ - $R$ color and constant continuum flux in the $R$-band wavelengths, so that the ratio of the band width of the $V$-band filter to the [O I] equivalent width corresponded to the ratio of the $V$-band luminosity to the [O I] luminosity. We used the formula of [16] for estimating the mass loss rate, $\dot{M}_{w}$,

$$
\log _{10} \dot{M}_{w}=-4.3+\log _{10} \frac{L_{6300}}{L_{\odot}},
$$




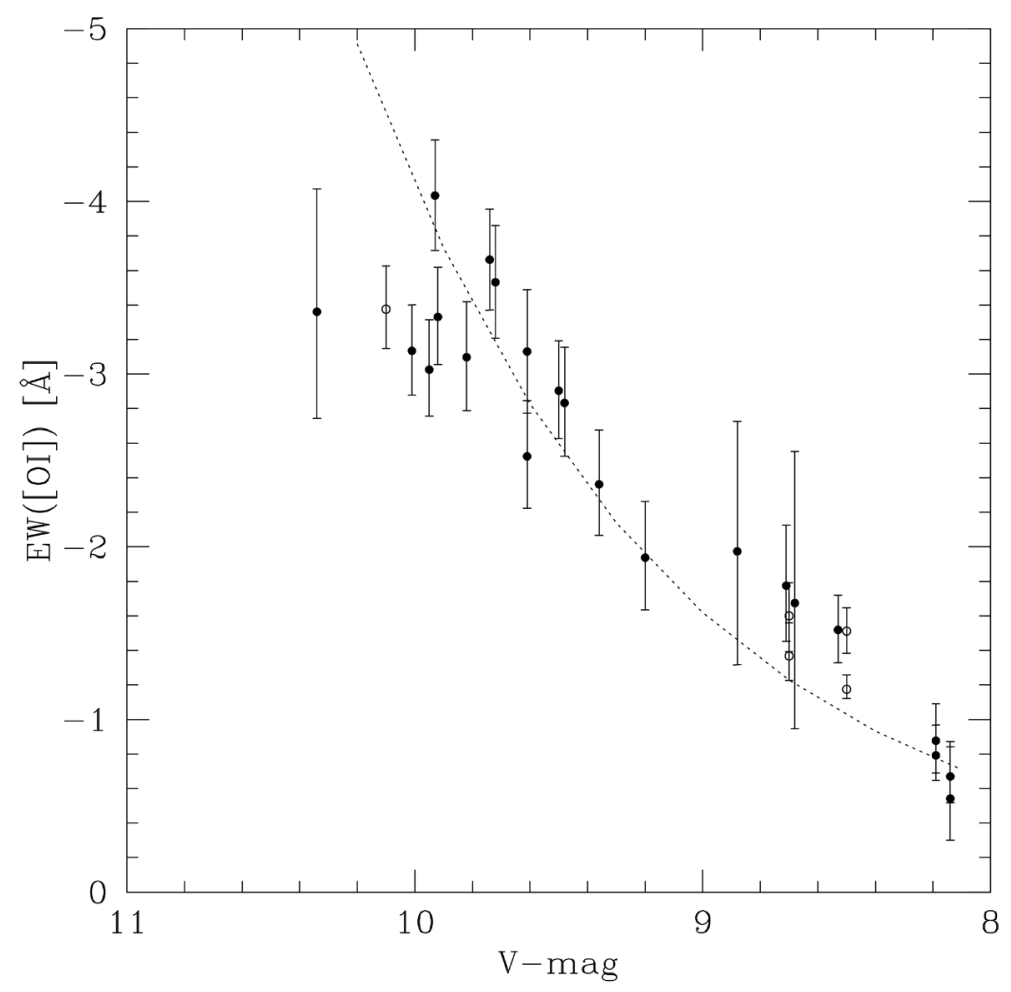

Figure 6. Relationship of the equivalent widths of the [O I] emission line and the $V$-band magnitudes. The filled circles indicate the MALLS data, and the open circles indicate the HIRES data. The equivalent widths decrease with an increase in the luminosity of the object. The dotted line shows the equivalent width of the [O I] line for the model in which the intensity of the emission line does not change as the continuum flux decreases. The model equivalent width is scaled so that it matches the observed equivalent width at $V=$ 9.6 mag. The variation in the equivalent widths of the [O I] emission line is almost consistent with the model.

where $L_{6300}$ is the [O I] luminosity and $L_{\odot}$ is the solar luminosity. The mass loss rate was found to be almost constant at $10^{-5.5} \mathrm{M}_{\odot} \cdot \mathrm{yr}^{-1}$.

The fact that the $[\mathrm{O} \mathrm{I}]$ emission line was unchanged in the luminosity and veiled by continuum light during the outbursts suggests that the origin of the jet is the FU Orionis object. Otherwise, the jet is insensitive to the rapid change in the mass accretion.

\section{3. $\mathrm{H} \alpha$}

$\mathrm{Z}$ CMa shows a strong $\mathrm{H} \alpha$ emission line. It is suggested that the $\mathrm{H} \alpha$ emission line of $\mathrm{Z} \mathrm{CMa} \mathrm{emanates} \mathrm{from} \mathrm{jet} \mathrm{and} \mathrm{that} \mathrm{its} \mathrm{high} \mathrm{velocity} \mathrm{component} \mathrm{comes}$ from the expanded atmosphere of the Herbig Be star [17]. We confirmed the P Cygni profile of the $\mathrm{H} \alpha$ line in all spectra (Figure 7). Figure 8 shows the relationship between the equivalent widths of the emission feature and the $V$-band magnitude of the object. We measured the equivalent widths by applying direct integration and did not measure the absorption feature. Figure 8 also shows the equivalent width of the $\mathrm{H} \alpha$ emission line for the model in which the continuum light component increases without any change in the intensity of the $\mathrm{H} \alpha$ line. 


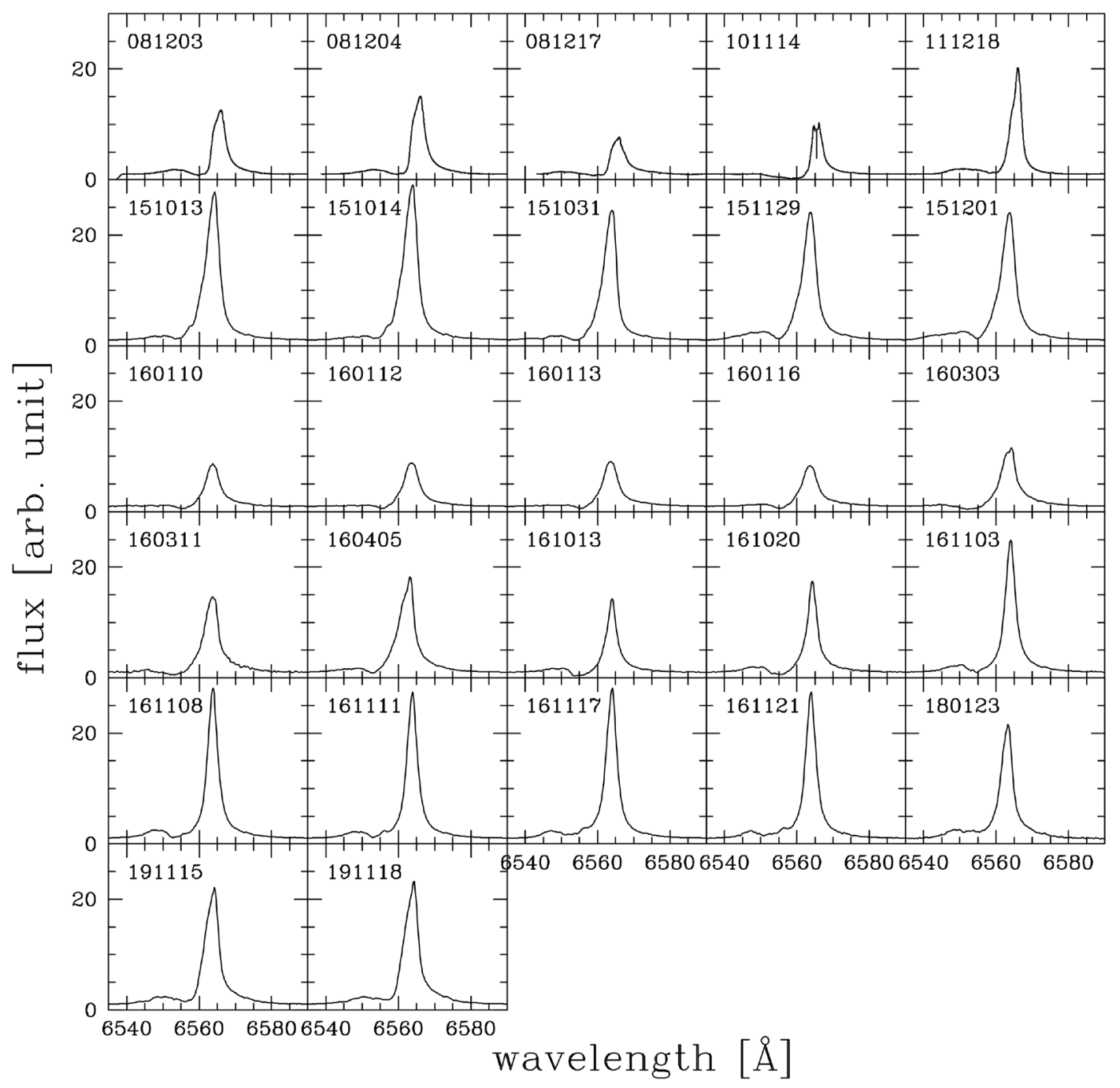

Figure 7. Line profiles of the $\mathrm{H} \alpha$ emission line.

The observed equivalent widths of the $\mathrm{H} \alpha$ line in the outburst phase seemed to be consistent with the model. $R^{2}$ is 0.91 between the model and the observed equivalent widths for $V<9.6$ mag. The $\mathrm{H} \alpha$ emission line was veiled to the continuum light when the outburst occurred. Based on the variation in the equivalent widths of the He I absorption line, it was determined that the Herbig Be star was the source of the outbursts. The fact that the $\mathrm{H} \alpha$ emission line was veiled with the continuum light suggests that the $\mathrm{H} \alpha$ line originates from a place unrelated to the outbursts, i.e., from the FU Orionis object. In the quiescent phase, the equivalent widths of the $\mathrm{H} \alpha$ emission line did not match the model. We discuss this equivalent width variation in the $\mathrm{H} \alpha$ line in the quiescent phase in the following sub-section.

\subsection{Fe II}

Several emission lines of Fe II were detected (Figure 9). The equivalent widths of three Fe II lines (6435 ̊̊, $6456 \AA$, and $6516 \AA$ ) were measured by employing 


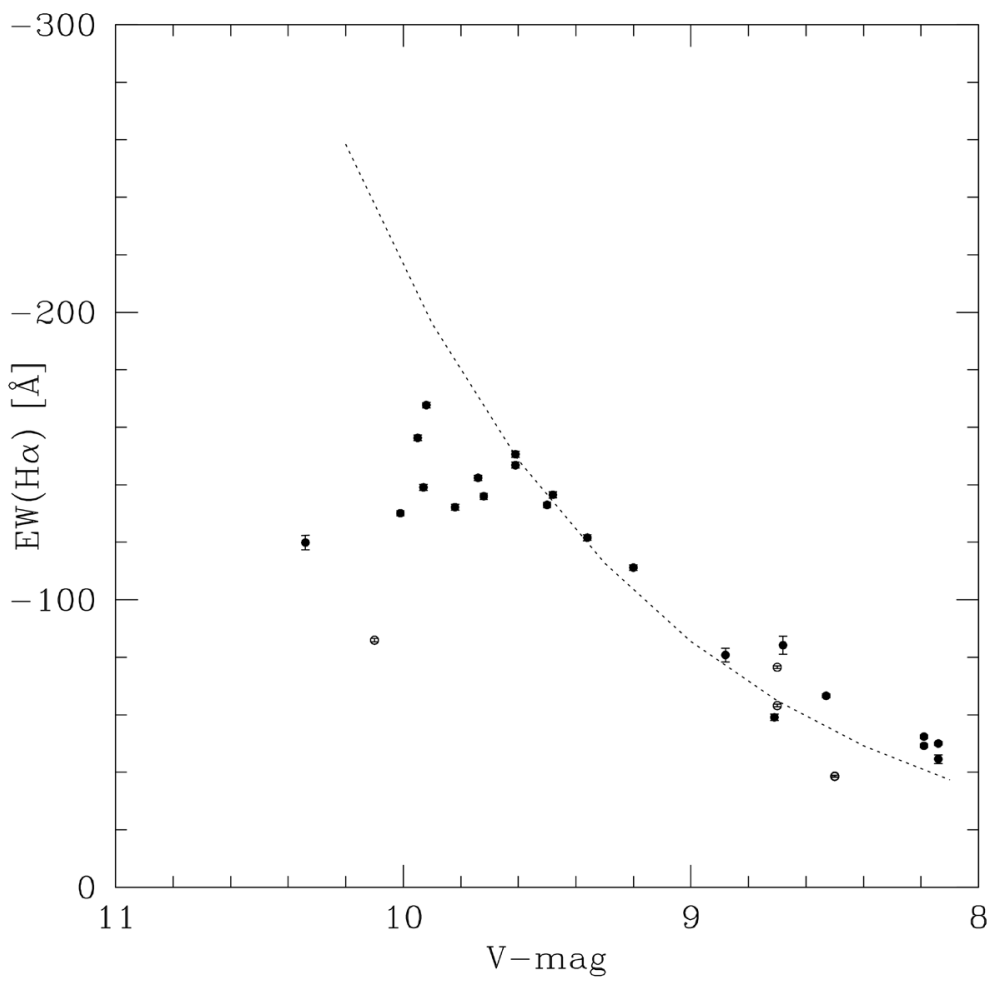

Figure 8. Relationship between the equivalent widths of the $\mathrm{H} \alpha$ emission line and the $V$-band magnitudes. The filled circles indicate the MALLS data. The open circles indicate the HIRES data. The dotted line shows the equivalent width for the model in which the intensity of the emission line does not change when the continuum flux decreases. The model equivalent width is scaled as it matches the observed equivalent width at $V=9.6$ mag. The variation in the $\mathrm{H} \alpha$ equivalent widths is almost consistent with the model. However, the observed equivalent widths do not match the model when the object was fainter than 9.6 magnitude in the $V$-band.

direct integration. Figure 10 shows the relationship between the equivalent widths of the three lines and the $V$-band magnitudes of the object. Figure 10 also shows the equivalent widths of the emission lines for the model in which the continuum light component increases without any change in the intensities of the Fe II lines. For $V<9.6 \mathrm{mag}, R^{2}$ between the model and the equivalent widths are $0.75,0.77$, and 0.70 for these lines, respectively. Our observations showed that the Fe II emission lines were veiled to the continuum light when the outbursts occurred. We considered that the lines originate from a place unrelated to the outbursts, i.e., from the FU Orionis object. In the quiescent phase, the intensities of the Fe II emission lines increased with an increase in the continuum flux.

\subsection{Origin of the Light Variations in the Outburst Phase and the Quiescent Phase}

In the previous sections, the equivalent widths of the emission lines of [O I] $\mathrm{H} \alpha$, and Fe II, as well as those of the He absorption feature, were investigated. In the outburst phase, the equivalent widths of the He I absorption line increased, 


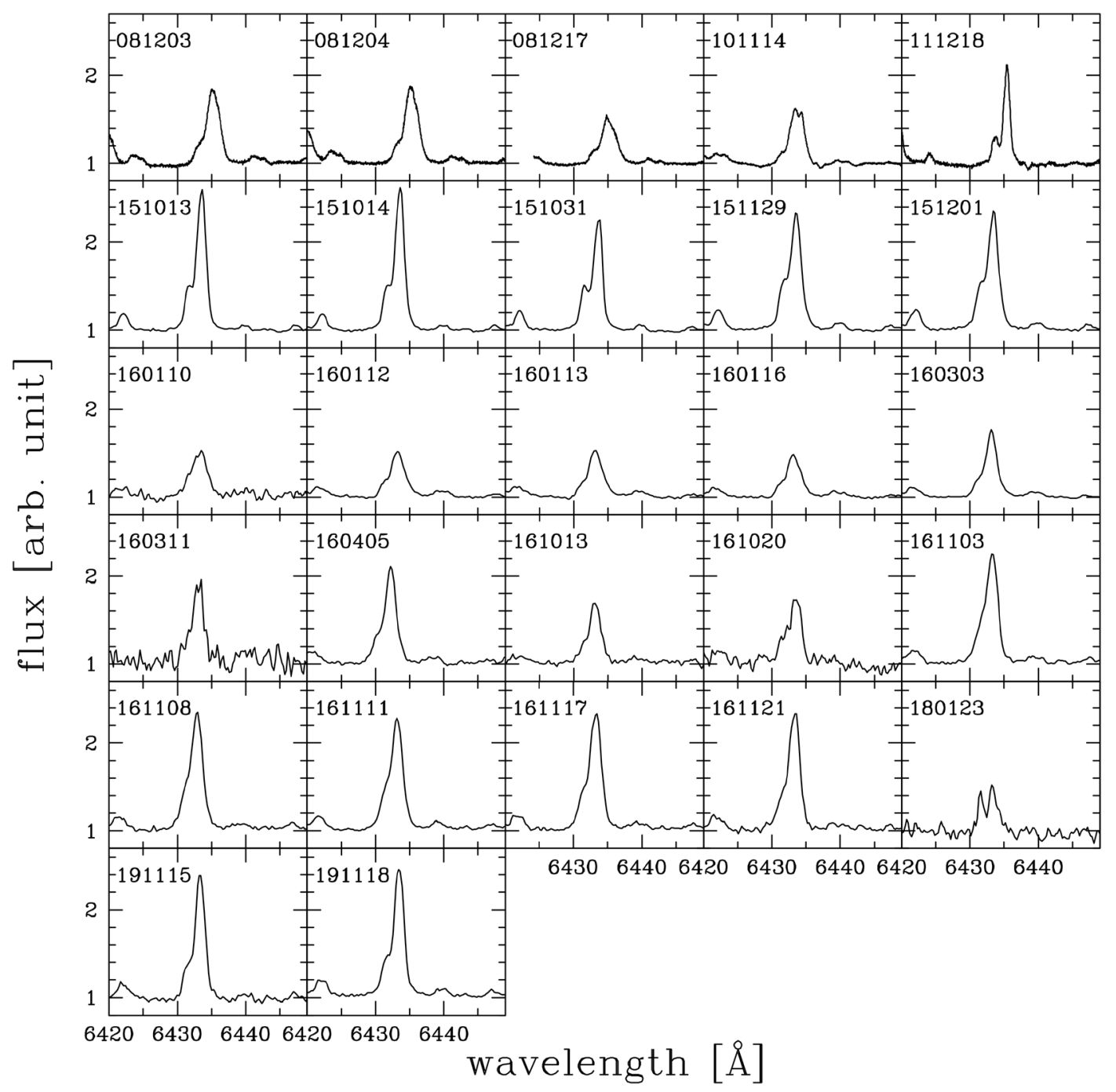

Figure 9. Line profile of the Fe II emission line at $\lambda 6435 \AA$.

whereas those of the [O I], $\mathrm{H} \alpha$, and Fe II emission lines decreased. We claimed that the Herbig Be star contributed to the optical continuum flux in the outburst phase. The $\mathrm{H} \alpha$ and Fe II emission lines were heavily veiled by the continuum light of the Herbig Be star in the outburst phase, and we think that these lines originated from the FU Orionis object. The [O I] line originated from a low-density region away from the central star such as a jet emanating from the FU Orionis object. Nature of the outburst is still under debate; it is not clear whether the outburst was originated as a consequence of a real outburst associated to the Herbig Be star, or because of changes in the dust cocoon such as the formation of a new hole. Broad-band photometry and polarimetric observations will reveal geometrical change of the cocoon, composition of the dust, and grain size distribution. If the amount of visible light transmitted through the cocoon changes, the amount of the visual extinction changes. Because the adjacent continuum flux as well as the line flux would change, an equivalent width of a line does not change. It is clear that the equivalent widths of many emission and 


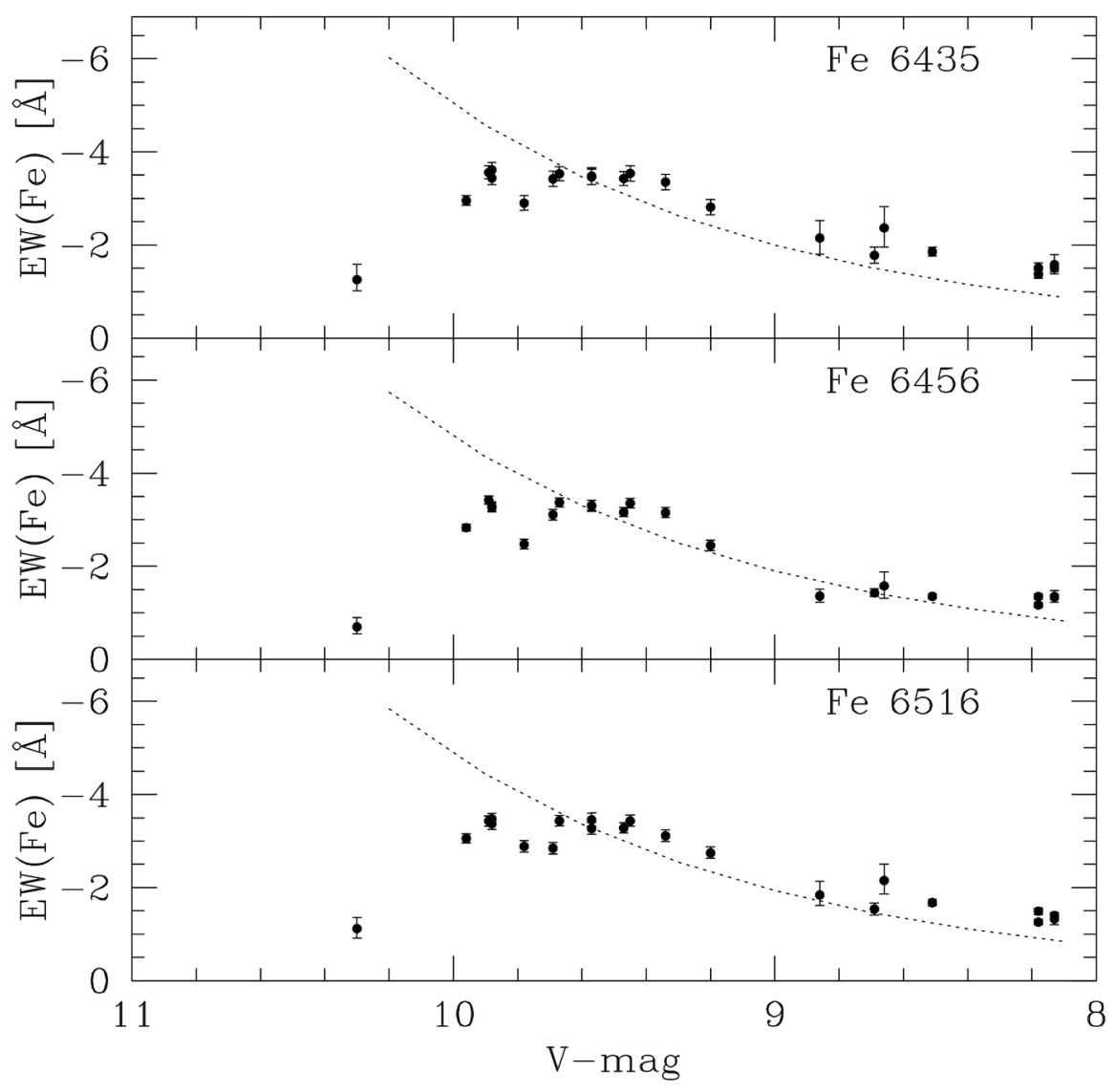

Figure 10. Relationship between the equivalent widths of the Fe II emission lines (6435 $\AA$, $6456 \AA$, and $6516 \AA$ ) and the $V$-band magnitudes. The dotted lines show the equivalent widths for the model in which the intensities of the emission lines do not change and the continuum flux decreases. The model equivalent widths are scaled as those match the observed equivalent widths at $V=9.6 \mathrm{mag}$. The Fe II equivalent widths are almost consistent with the model. However, the observed equivalent widths do not match the model when the object was fainter than 9.6 magnitude in the $V$-band.

absorption lines changed during the outbursts. These phenomena are not accounted by the geometrical change of the dust cocoon. We claim that, at least, a part of the outbursts are attributed to the real outburst of the Herbig Be star.

The equivalent widths of the emission and absorption lines also changed in the quiescent phase. The He I absorption feature was barely detected. The [O I] emission line was veiled by the continuum light. The intensities of the $\mathrm{H} \alpha$ and $\mathrm{Fe}$ II emission lines increased as the star became brighter. Previous studies suggested that the FU Orionis object dominates the $V$-band luminosity during the quiescent phase [11]. We considered that the $\mathrm{H} \alpha$ and Fe II emission lines originated from the FU Orionis object. Because the FU Orionis object showed the emission lines even in the quiescent phase, we considered that the light variation in the quiescent phase was caused by mass accretion to the FU Orionis object. We propose that the light variation in the outburst phase is due to the Herbig Be star and the light variation in the quiescent phase is caused by the FU Orionis object. 


\section{Conclusion}

We conducted optical spectroscopic monitoring observations of a young binary Z CMa. Medium-resolution spectra were obtained on 21 nights between 2015 and 2019. During the observation period, the object experienced four brightening phases with an amplitude of 2 magnitudes in the $V$-band. The intensity of the He I absorption line increased with an increase in the luminosity of the object. Because the He I line is a characteristic feature of an early-type star, we concluded that large-amplitude light variations were associated with the Herbig Be star. The equivalent widths of the [O I] line decreased as the continuum flux increased. The mass loss rate estimated from the equivalent widths of the [O I] line remained almost constant at $10^{-5} \mathrm{M}_{\odot} \cdot \mathrm{yr}^{-1}$. The intensities of the $\mathrm{H} \alpha$ and $\mathrm{Fe}$ II emission lines did not change in the outburst phase. However, these lines became stronger as the luminosity of the object increased in the quiescent phase. We propose that the light variations in the outburst phase are caused by the Herbig Be star and those in the quiescent phase are due to the FU Orionis object. Because the FU Orionis object shows emission lines, we claim that its light variation is caused by mass accretion.

\section{Acknowledgements}

This work was supported by JSPS KAKENHI Grant Number JP17K05390.

\section{Conflicts of Interest}

The authors declare no conflicts of interest regarding the publication of this paper.

\section{References}

[1] Muzerolle, J., Hartmann, L. and Calvet, N. (1998) A Br $\gamma$ Probe of Disk Accretion in T Tauri Stars and Embedded Young Stellar Objects. The Astronomical Journal, 116, 2965-2974. https://doi.org/10.1086/300636

[2] Kenyon, S.J. and Hartmann, L.W. (1991) The Dusty Envelopes of FU Orionis Variables. Astrophysical Journal, 383, 664-673. https://doi.org/10.1086/170823

[3] van den Ancker, M.E., Blondel, P.F.C., Tjin A Djie, H.R.E., Grankin, K.N., Ezhkova, O.V., Shevchenko, V.S., et al. (2004) The Stellar Composition of the Star Formation Region CMa R1-III. A New Outburst of the Be Star Component in Z CMa. Monthly Notices of the Royal Astronomical Society, 349, 1516-1536. https://doi.org/10.1111/j.1365-2966.2004.07629.x

[4] Szeifert, T., Hubrig, S., Schöller, M., Schütz, O., Stelzer, B. and Mikulásek, Z. (2010) The Nature of the Recent Extreme Outburst of the Herbig Be/FU Orionis Binary Z Canis Majoris. Astronomy \& Astrophysics, 509, Article No. L7. https://doi.org/10.1051/0004-6361/200913704

[5] Bonnefoy, M., Chauvin, G., Dougados, C., Kóspál, Á., Benisty, M., Duchêne, G., et al. (2017) The 2008 Outburst in the Young Stellar System Z CMa. III. Multi-Epoch High-Angular Resolution Images and Spectra of the Components in Near-Infrared. Astronomy \& Astrophysics, 597, Article No. A91.

https://doi.org/10.1051/0004-6361/201628693 
[6] Koresko, C.D., Beckwith, S.V.W., Ghez, A.M., Matthews, K. and Neugebauer, G. (1991) An Infrared Companion to Z Canis Majoris. Astronomical Journal, 102, 2073-2078. https://doi.org/10.1086/116031

[7] Haas, M., Christou, J.C., Zinnecker, H., Ridgway, S.T. and Leinert, Ch. (1993) Sub-Diffraction-Limited Infrared Speckle Observations of Z Canis, a 0".10 Variable Binary Star. Astronomy \& Astrophysics, 269, 282-290.

[8] Hartmann, L., Kenyon, S.J., Hewett, R., Edwards, S., Strom, K.M., Strom, S.E., et al. (1989) Pre-Main-Sequence Disk Accretion in Z Canis Majoris. Astrophysical Journal, 338, 1001-1010. https://doi.org/10.1086/167252

[9] Whitney, B.A., Clayton, G.C., Schulte-Ladbeck, R.E., Calvet, N., Hartmann, L. and Kenyon, S.J. (1993) Spectrum of the "Invisible" Companion of Z Canis Majoris Revealed in Polarized Light. Astrophysical Journal, 417, 687-696.

https://doi.org/10.1086/173347

[10] Canovasm, H., Min, M., Jeffers, S.V., Rodenhuis, M. and Keller, C.U. (2012) Constraining the Circumbinary Envelope of $\mathrm{Z}$ Canis Majoris via Imaging Polarimetry. Astronomy \& Astrophysics, 543, Article No. A70. https://doi.org/10.1051/0004-6361/201117762

[11] Hinkley, S., Hillenbrand, L., Oppenheimer, B.R., Rice, E.L., Pueyo, L., Vasisht, G., et al. (2013) High-Resolution Infrared Imaging and Spectroscopy of the Z Canis Majoris System during Quiescence and Outburst. The Astrophysical Journal, 763, Article No. L9. https://doi.org/10.1088/2041-8205/763/1/L9

[12] Hessman, F.V., Eisloeffel, J., Mundt, R., Hartmann, L.W., Herbst, W. and Krautter, J. (1991) The High State of the FU Orionis Variable Z Canis Majoris. Astrophysical Journal, 370, 384-395. https://doi.org/10.1086/169824

[13] Maehara, H. (2014) Automated Wide-Field Survey for Transient Objects with a Small Telescope. Journal of Space Science Informatics Japan, 3, 119-127.

[14] Hamann, F. and Persson, S.E. (1989) High-Resolution Spectra of the Luminous Young Stellar Object V645 Cygni. Astrophysical Journal, 339, 1078-1088. https://doi.org/10.1086/167362

[15] Antoniucci, S., Podio, L., Nisini, B., Bacciotti, F., Lagadec, E., Sissa, E., et al. (2016) Sub-0.1" Optical Imaging of the Z CMa Jets with SPHERE/ZIMPOL. Astronomy \& Astrophysics, 593, Article No. L13. https://doi.org/10.1051/0004-6361/201628968

[16] Hartigan, P., Edwards, S. and Ghandour, L. (1995) Disk Accretion and Mass Loss from Young Stars. Astrophysical Journal, 452, 736-768.

https://doi.org/10.1086/176344

[17] Bailey, J. (1998) Detection of Pre-Main-Sequence Binaries Using Spectro-Astrometry. Monthly Notices of the Royal Astronomical Society, 301, 161-167. https://doi.org/10.1046/j.1365-8711.1998.02010.x 